\title{
An historical and contemporary review of endovascular therapy for acute ischemic stroke
}

\author{
Karl Boyle ${ }^{1 *}$, Raed A. Joundi ${ }^{1}$ and Richard I. Aviv ${ }^{2}$
}

\begin{abstract}
Long standing, evidence based approved therapies for acute ischemic stroke include intravenous thrombolysis therapy (IVT) with alteplase (recombinant tissue plasminogen activator, rtPA) given within 4.5 h; aspirin therapy within 48 h; management in an acute stroke unit and hemicraniectomy in cases of malignant infarction. Multiple recent positive randomized controlled trials (RCTs) have now also established endovascular therapy with mechanical thrombectomy as the standard of care for acute ischemic stroke involving a large vessel occlusion in the anterior circulation.

This article will review the history of endovascular treatments for acute ischemic stroke and will review the recent positive and negative randomized controlled trial evidence for its efficacy. Current guidelines and dilemmas regarding appropriate patient selection will be discussed.
\end{abstract}

Keywords: Thrombolysis, Endovascular, Imaging, Stroke, Trials, CT, Perfusion

\section{Background}

IVT is the standard of care for acute ischemic stroke [1-3]. Yet IVT must be delivered rapidly within $4.5 \mathrm{~h}$, and has many contraindications. Furthermore, many patients with large or proximal clots may not achieve adequate reperfusion with thrombolysis, and treatment carries the risk of intracranial haemorrhage, which can be fatal.

As a result, there have been a wide variety of dedicated trials over the past 20 years to treat proximal clots not responsive to thrombolysis with interventional and mechanical means. As reviewed below, early trials in endovascular therapy did not demonstrate a benefit in patients with acute ischemic stroke, whereas newer trials have demonstrated substantial efficacy in the treatment of proximal occlusions producing a major breakthrough for treatment options in hyperacute ischemic stroke.

\footnotetext{
* Correspondence: Karl.Boyle@sunnybrook.ca

${ }^{1}$ Department of Medicine, Division of Neurology, Sunnybrook Health Science Centre and University of Toronto, 2075 Bayview Avenue, Toronto M4N 3M5, Canada

Full list of author information is available at the end of the article
}

\section{Early experience with endovascular treatment in acute stroke Intra-arterial thrombolysis (IAT)}

The PROACT trials introduced the initial promise of intra-arterial treatment for ischemic stroke $[4,5]$. PROACT I demonstrated IAT administration of $6 \mathrm{mg}$ pro-urokinase in patients with M1 or M2 occlusions resulted in higher recanalization rates, although all patients received intravenous heparin, and clinical outcomes were not measured. In PROACT II, patients with angiographically proven proximal MCA occlusions were randomized to receive IAT with $9 \mathrm{mg}$ of pro-urokinase (given proximal to the clot and mechanical clot disruption with the guide wire was not allowed) plus heparin or heparin only in the control arm. Pro-urokinase administration within $6 \mathrm{~h}$ resulted in a significantly higher number of patients with modified Rankin scale (mRS) score of 2 or less at 90 days $(40 \% \mathrm{v} 25 \%)$, with recanalization rates of $66 \%$ v $18 \%$. Symptomatic intracranial haemorrhage occurred in $10 \%$ versus $2 \%$ in controls and mortality was $25 \%$ versus $27 \%$ in controls. Pro-urokinase was never approved by the FDA for this indication, citing the need for a confirmatory trial that was never performed. 


\section{First generation thrombectomy devices}

Following the results of the 1999 PROACT II trial, the MERCI retriever device was developed. This was the first generation of mechanical thrombectomy devices approved by the FDA, and propelled an era of interventional stroke trials. MERCI devices were found to achieve recanalization rates (defined as thrombolysis in myocardial infarction score (TIMI) 2 or 3 flow in internal carotid artery (ICA), M1 and M2 (first and second portion of the middle cerebral artery (MCA) branches) of $46 \%$ in the first MERCI trial and $55 \%$ in the follow up MULTI MERCI trial [6, 7]. Good clinical outcome (modified Ranking Scale, $\mathrm{mRS} \leq 2$ ) was 28 and $36 \%$ in the two trials but mortality remained high (44 and 34\% respectively). However both trials, which treated patients within $8 \mathrm{~h}$, were single arms trials compared to historical controls. Nevertheless, patients with successful recanalization were more likely to achieve good clinical outcome, establishing a clinical rationale for early recanalization after acute stroke.

\section{Second generation thrombectomy devices}

The results of a second generation device, the Penumbra aspiration system, were reported in the Penumbra Pivotal Stroke Trial [8]. This trial was not randomized but rather the goal of the trial was to provide substantial equivalence in safety and effectiveness to the MERCI device in opening occluded blood vessels in stroke. High vessel revascularization rates (defined as TIMI 2 or 3 flow at the site of primary occlusion only) of $82 \%$ were achieved compared to those reported for the MERCI device. However clinical outcomes remained poor with only $25 \%$ achieving a good clinical outcome $(\mathrm{mRS} \leq 2)$ and high all-cause mortality rate of $33 \%$. Complication rates were high with $12.8 \%$ of patients experiencing a procedural complication, of which $2.4 \%$ were considered serious, and a $24 \mathrm{~h}$ intracranial haemorrhage $(\mathrm{ICH})$ rate of $28 \%$ with a symptomatic ICH rate of $11.2 \%$. Similar to the MERCI trials, an $8 \mathrm{~h}$ time window was used.

\section{Third generation thrombectomy devices}

The SOLITAIRE and Trevo devices were both retrievable stents, a technology that continued the evolution of thrombectomy device. Promising recanalization rates and clinical outcomes, as compared to the Merci device, were reported in the SWIFT and TREVO 2 trials, heralding what was to come in later RCTs $[9,10]$.

2013 - the year of three negative EVT RCTs (Tables 1, 2, 3 and 4) In March 2013, three randomized controlled trials, SYNTHESIS, MR RESCUE, and IMS III, were presented at the International Stroke Conference in Hawaii and subsequently published in the same issue of the New
England Journal of Medicine. Very disappointingly all three trials reported negative results [11-13].

Using the MERCI retriever or PENUMBRA system, MR RESCUE, completed over an 8 year period, compared patients receiving endovascular therapy versus those receiving standard care. Inclusion criteria included demonstration of intracranial ICA or M1 occlusion, NIHSS greater or equal to 6 and once again the eligible time window was within $8 \mathrm{~h}$. Forty-four percent received tPA in the endovascular arm compared to $30 \%$ in the standard care arm. There was no outcome difference between groups, irrespective of whether the patient had a favourable penumbral pattern or nonpenumbral pattern based on perfusion imaging. Reperfusion, defined as modified thrombolysis in cerebral infarction score (mTICI) $2 \mathrm{a} / 3$ was $67 \%$ and defined as mTICI $2 \mathrm{~b} / 3$ was only $27 \%$. Good clinical outcome (mRS $0-2$ ) was only demonstrated in $14 \%$. Older generation devices were used and the mean time to endovascular procedure was $6 \mathrm{~h}$ and $21 \mathrm{~min}$.

IMS III was a large, randomized controlled trial comparing endovascular therapy plus IVT (IVT stopped at $40 \mathrm{~min}$ ) to IVT alone. Demonstration of intracranial occlusion was not required. In IMS III, National Institutes of Health Stroke Scale, NIHSS $\geq 10$ was used as a marker of stroke severity and risk of proximal occlusion, but as Computed Tomography Angiography, CTA became more widespread, an amendment mid-way through the trial allowed screening for proximal clots with CTA for patients with NIHSS of 8 or 9. MERCI, PENUMBRA, SOLITAIRE, or Microcatheter delivery of intra-arterial tPA was allowed. As a result, the methods were a mix of pharmacological thrombolysis, manipulation of clot with use of a guidewire or microcatheter, mechanical and aspiration thrombectomy, and stent-retriever technology. The procedure was required to begin within $5 \mathrm{~h}$. Disappointingly, there was no difference in outcome between groups with $41 \%$ good clinical outcome (mRS 0-2) in the combined IVT/EVT arm versus $39 \%$ in the IVT only arm. Recanalization rate (defined as modified arterial occlusion lesion score mAOL 2-3) was $81 \%$ for ICA occlusion and $86 \%$ for $\mathrm{M} 1$ occlusion; the reperfusion rate (mTICI $2 \mathrm{~b} / 3$ ) was $38 \%$ for ICA occlusion and $44 \%$ for M1 occlusion. Retrievable stents were only used in 14 patients.

The SYNTHESIS trial compared endovascular therapy (intra-arterial thrombolysis with rtPA, mechanical clot disruption or retrieval or a combination of these approaches) to treatment with intravenous tPA alone. Demonstration of vessel occlusion prior to endovascular treatment was not required nor was any clinical severity rating on the NIHSS. Reperfusion rates were not reported. There was no difference in good clinical outcome $-42 \%$ in the endovascular arm versus $46 \%$ in the intravenous tPA arm. A mechanical device was only 


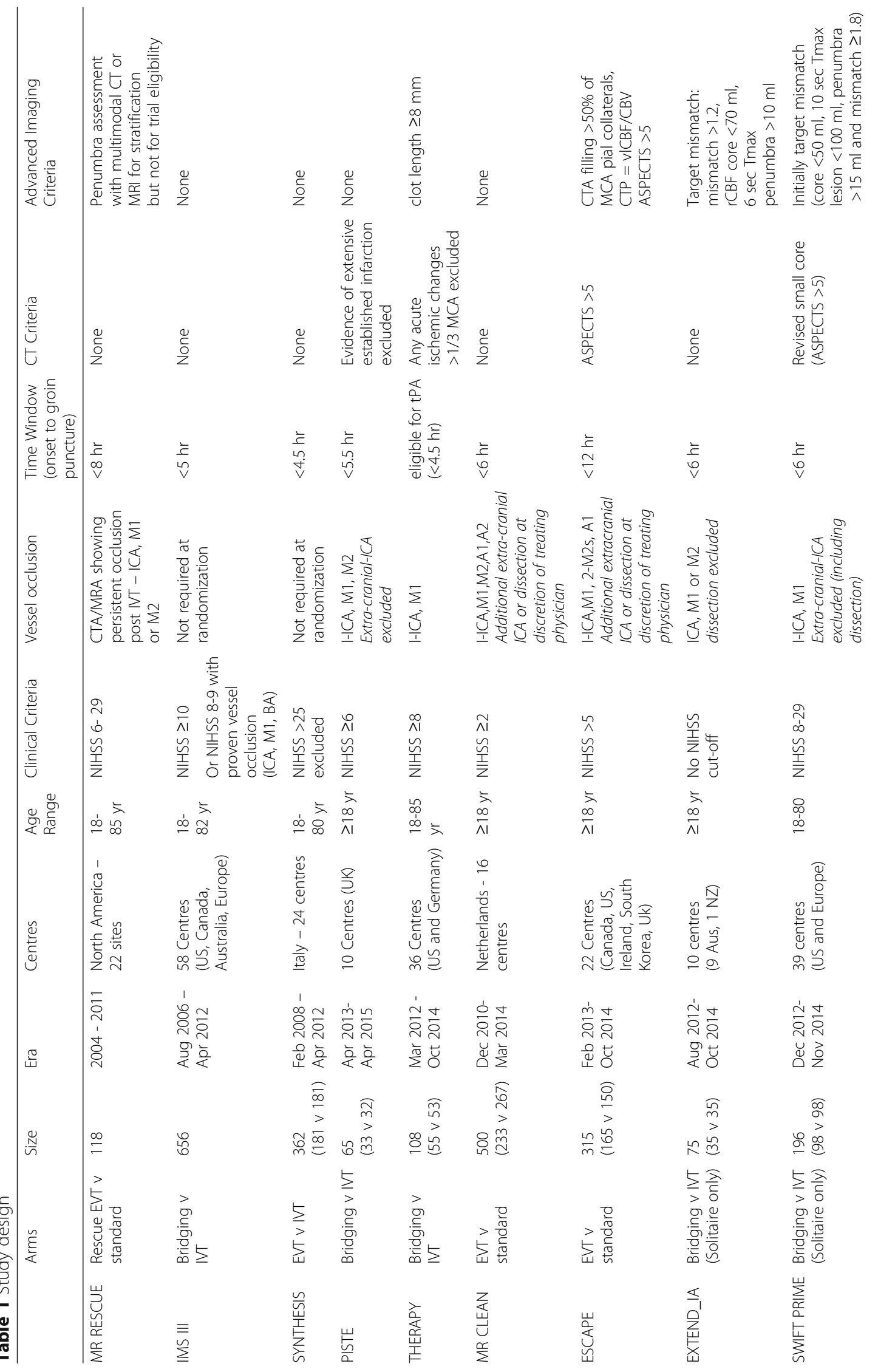




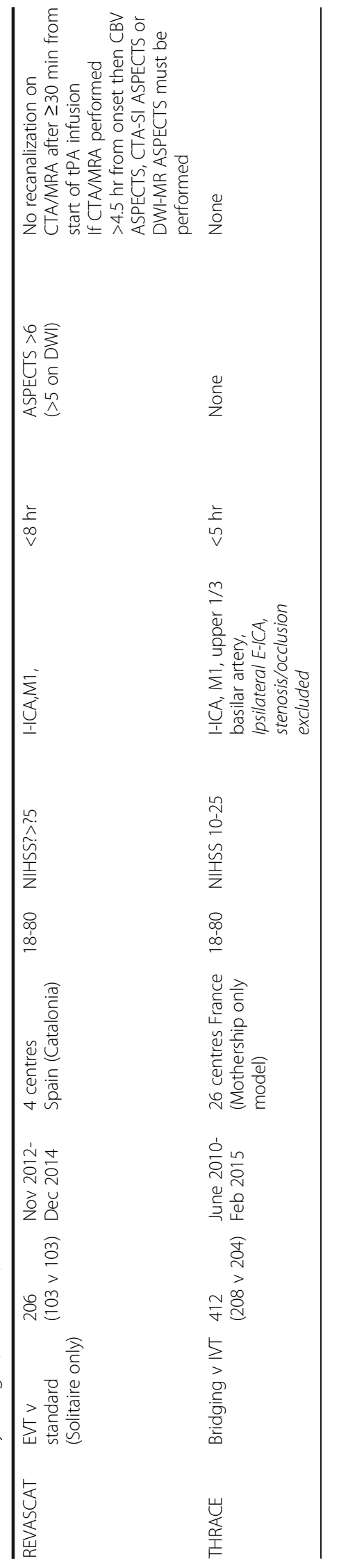


Table 2 Baseline characteristics

\begin{tabular}{|c|c|c|c|c|c|c|c|c|}
\hline & Age (median) & Male (\%) & $\begin{array}{l}\text { NIHSS } \\
\text { (median) }\end{array}$ & Vessel occlusion & $\begin{array}{l}\text { Tandem lesion } \\
\text { (extrancranial } \\
\text { ICA occlusion) }\end{array}$ & ASPECTS (median) & IVT (\%) & $\begin{array}{l}\text { Retrievable } \\
\text { stent (\%) }\end{array}$ \\
\hline MR RESCUE & 66 & 50 & 16 & $71 \%$ ICA or $\mathrm{M}^{*}$ & $\mathrm{nr}$ & $\begin{array}{l}\text { predicted core } \\
36 \mathrm{ml}^{*}\end{array}$ & $47^{*}$ & 0 \\
\hline |MS ||| & 69 & 50 & 17 & $\begin{array}{l}18 \% \text { of EVT group } \\
\text { had no occlusion }\end{array}$ & $\mathrm{nr}$ & $\mathrm{nr}$ & 100 & 4 \\
\hline SYNTHESIS & 66 & 59 & 13 & $2 \%$ no occlusion & $\mathrm{nr}$ & $\mathrm{nr}$ & 0 & 41 \\
\hline PISTE & 67 & 39 & 18 & $\begin{array}{l}90 \% \text { carotid T/L } \\
\text { or } \mathrm{M} 1\end{array}$ & $3 \%$ & 9 & 100 & 68 \\
\hline THERAPY & 67 & 62 & 17 & $89 \%$ I-ICA or M1 & excluded & 7.5 & 100 & $\begin{array}{l}\text { 13\% (majority } \\
\text { used aspiration } \\
\text { thrombectomy) }\end{array}$ \\
\hline MR CLEAN & 66 & 58 & 17 & $\begin{array}{l}92 \% \text { I-ICA, carotid } \\
\text { T or M1 }\end{array}$ & $32 \%$ & 9 & $\begin{array}{l}87 \\
\text { (44\% drip \& ship) }\end{array}$ & 82 \\
\hline ESCAPE & 71 & $\begin{array}{l}48(87 \% \\
\text { white) }\end{array}$ & 16 & $\begin{array}{l}96 \% \text { carotid } \mathrm{T} / \mathrm{L} \\
\text { or } \mathrm{M} 1\end{array}$ & $13 \%$ & 9 & 73 & 73 \\
\hline EXTEND_IA & 69 & 49 & 17 & $88 \%$ I-ICA or M1 & $n / r$ & $\begin{array}{l}\mathrm{n} / \mathrm{r} \\
\text { (median core } 12 \mathrm{ml} \text { ) }\end{array}$ & 100 & 100 \\
\hline SWIFT PRIME & 65 & $\begin{array}{l}55(89 \% \\
\text { white) }\end{array}$ & 17 & $\begin{array}{l}86 \% \text { carotid T/L } \\
\text { or } \mathrm{M} 1\end{array}$ & excluded & 9 & $\begin{array}{l}100 \\
\text { (44\% drip \& ship) }\end{array}$ & 100 \\
\hline REVASCAT & 65 & 55 & 17 & $\begin{array}{l}90 \% \text { carotid T/L } \\
\text { or } \mathrm{M} 1\end{array}$ & 19 & 7 & 70 & 70 \\
\hline THRACE & 66 & 57 & 18 & $98 \%$ ICA or $\mathrm{M} 1$ & excldued & $\mathrm{nr}$ & $\begin{array}{l}100 \\
\text { (100\% mothership) }\end{array}$ & 77 \\
\hline
\end{tabular}

${ }^{\mathrm{a} M R}$ RESCUE values reported for penumbral group receiving embolectomy

used in 56/181 patients randomized to endovascular treatment; however, this trial did see the introduction of the third generation of mechanical devices, the retrievable stents, which were used in 23/56 patients in whom a device was deployed.

Overall, these three trials failed to show a benefit for endovascular intervention in ischemic stroke. However, a variety of limitations were identified and addressed in the design of new endovascular therapy RCTs [14, 15]. Firstly, the use of non-invasive angiography was not universal in patient selection. For example, in IMS III, more than 50\% of patients did not undergo CTA, as it was not in widespread use during early patient recruitment. In MR RESCUE, patients were eligible only if angiography showed persistent target occlusion after receiving tPA. Secondly, there were long time delays from stroke onset to

Table 3 Process times

\begin{tabular}{|c|c|c|c|c|c|c|c|c|}
\hline & $\begin{array}{l}\text { Onset to IVT } \\
\text { (median., min) }\end{array}$ & $\begin{array}{l}\text { Onset to } \\
\text { randomisation } \\
\text { (median., min) }\end{array}$ & $\begin{array}{l}\text { Onset to groin } \\
\text { puncture } \\
\text { (median., min) }\end{array}$ & $\begin{array}{l}\text { Onset to first } \\
\text { reperfusion } \\
\text { (median., min) }\end{array}$ & $\begin{array}{l}\text { Groin puncture } \\
\text { to reperfusion } \\
\text { (median., min) }\end{array}$ & $\begin{array}{l}\text { IVT to groin } \\
\text { puncture } \\
\text { (median., min) }\end{array}$ & $\begin{array}{l}\text { CT to groin } \\
\text { puncture } \\
\text { (median., min) }\end{array}$ & $\begin{array}{l}\text { CT to reperfusion } \\
\text { (median., min) }\end{array}$ \\
\hline MR RESCUE & $\mathrm{nr}$ & $\mathrm{nr}$ & 381 & $\mathrm{nr}$ & $\mathrm{nr}$ & $\mathrm{nr}$ & 124 & $\mathrm{nr}$ \\
\hline IMS III & 122 & $\mathrm{nr}$ & 208 & $\mathrm{nr}$ & $\mathrm{nr}$ & $\mathrm{nr}$ & $\mathrm{nr}$ & $\mathrm{nr}$ \\
\hline SYNTHESIS & 165 & 148 & 225 & $\mathrm{nr}$ & $\mathrm{nr}$ & $\mathrm{nr}$ & $\mathrm{nr}$ & $\mathrm{nr}$ \\
\hline PISTE & 120 & 150 & 209 & 259 & $49^{*}$ & 82 & $58 \#$ & $\mathrm{nr}$ \\
\hline THERAPY & 108 & 181 & 227 & $\mathrm{nr}$ & $\mathrm{nr}$ & $\mathrm{nr}$ & 123 & $\mathrm{nr}$ \\
\hline MR CLEAN & 85 & 204 & 260 & 332 & $\mathrm{nr}$ & $\mathrm{nr}$ & $\mathrm{nr}$ & $\mathrm{nr}$ \\
\hline ESCAPE & 110 & 169 & 208 & 241 & 30 & 51 & 51 & 84 \\
\hline EXTEND_IA & 127 & 156 & 210 & 248 & 43 & 74 & 93 & $\mathrm{nr}$ \\
\hline SWIFT PRIME & 111 & 191 & 224 & 252 & $\mathrm{nr}$ & $\mathrm{nr}$ & 58 & 87 \\
\hline REVASCAT & 118 & 223 & 269 & 355 & 59 & $\mathrm{nr}$ & 67 & $\mathrm{nr}$ \\
\hline THRACE & 150 & 168 & 250 & $\mathrm{nr}$ & $\mathrm{nr}$ & $\mathrm{nr}$ & $\mathrm{nr}$ & $\mathrm{nr}$ \\
\hline
\end{tabular}

${ }^{\mathrm{a}}$ Groin puncture to device removal

${ }^{\mathrm{b}}$ Randomisation to groin puncture 


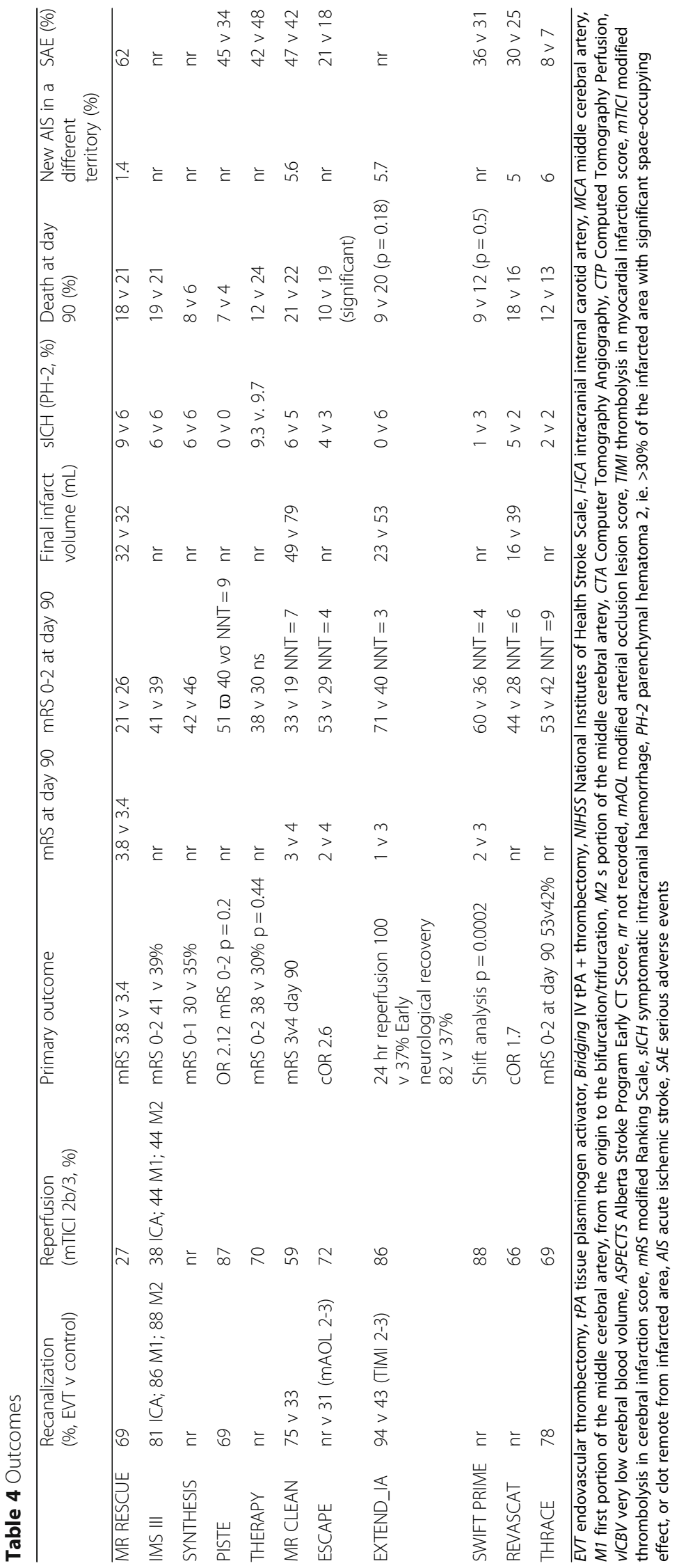


revascularization, in part due to lack of rapid workflow. Lastly, devices were relatively limited in their ability to achieve recanalization and the new generation retrievable stents were used in a small number of patients.

\section{Recent landmark positive RCTs demonstrating efficacy and safety of EVT (Tables 1, 2, 3 and 4)}

In 2015 the landscape completely changed with the publication of five randomized controlled trials showing positive results for endovascular treatment in patients with acute ischemic stroke. presenting with large vessel occlusion in the anterior circulation [16-20]. A sixth positive trial has been published in 2016 [21]. These six trials focused on the previous deficiencies in order to maximize work-flow and patient selection, by 1) mandating universal vascular imaging to identify patients with proximal occlusion 2) emphasizing rapid door-torecanalization times, and 3) use of advanced retrievable stent technology.

The first landmark positive trial was MR CLEAN, presented at the $9^{\text {th }}$ World Stroke Congress in October 2014. The trial recruited patients within $6 \mathrm{~h}$ of onset, with proximal occlusion of the anterior circulation (distal ICA, M1 or M2, first or second portion of Anterior cerebral artery, A1 or A2), and NIHSS $>2$. This was a very pragmatic trial conducted within a single country (the Netherlands) and it is important to note that neither the basic non contrast Computed Tomography, NCCT brain Alberta Stroke Program Early CT Score, (ASPECTS score) nor advanced clinical imaging (collateral scoring or perfusion imaging) were used to identify and exclude patients with large core infarction. The vast majority of patients received IVT (89\%) and $82 \%$ of patients were treated with retrievable stents. This trial shifted from using a dichotomized mRS as the primary outcome to using the adjusted common odds ratio for a shift in the direction of a better outcome on the mRS. The adjusted common odds ratio was 1.67 (95\% CI 1.21 to 2.30), representing the first positive RCT for endovascular therapy. Statistically significantly more patients achieved functional independence (mRS 0 to 2) at 90 days in the intervention group, 33\% compared to $19 \%$ (95\% CI 5.9 to 21.2 ) with an adjusted odds ratio of 2.16 (95\% CI 1.39 to 3.38 ).

Following early interim analyses prompted by these positive results, five other ongoing RCTs stopped recruitment early and swiftly produced concordant results. MR CLEAN therefore remains the only fully powered study. Unpowered studies may overestimate effect size but it is reassuring that the same positive result was obtained in five different prematurely terminated RCTs. Those trials will now be discussed.

The ESCAPE trial, conducted February 2013 to October 2014 across 22 sites in Canada, United States,
Ireland and South Korea, allowed recruitment of patients within $12 \mathrm{~h}$, the longest time window of all the trials, with clinical severity requirements for inclusion set at a NIHSS $>6$. Additional advanced imaging criteria were also required: (i) NCCT ASPECTS $>5$ aimed at identifying small core infarcts; (ii) proximal intracranial occlusion of M1, M2, or intracranial ICA was required on vascular imaging, with tandem occlusion of the extra-cranial internal carotid artery also allowed; and (iii) moderate to good collaterals, defined as filling of $>50 \%$ of MCA pial arterial circulation on CTA, preferably acquired with multiphase CTA. Although not obligatory, if CT perfusion was used, a low CBV or very low CBF ASPECTS $>5$ was needed. This trial also mandated an imaging-to-groin puncture time of $<60 \mathrm{~min}$ and a target groin puncture to reperfusion time of $<30 \mathrm{~min}$. Wake up strokes and patients ineligible for intravenous tPA were also accepted if the above criteria were fulfilled. Rapid workflow was emphasized, thus achieving the shortest onset-to-reperfusion time among the trials - the median stroke onset to reperfusion was $4 \mathrm{~h}$. Seventy-six percent of patients received IVT. The trial demonstrated an increase in functional independence (mRS $0-2)$ at 90 days from $29 \%$ in the control group to $53 \%$ in the intervention group $(p<0.001)$ and the primary outcome favoured the intervention with a common odds ratio of 2.6 (95\% CI 1.7 to 3.8; $p<0.001)$. ESCAPE was the only trial to demonstrate a statistically significant reduction in mortality from 19 to $10 \%(p=0.04)$.

The Australian and New Zealand EXTEND-IA trial, conducted from August 2012 through October 2014 across ten sites, had the most stringent selection criteria and was the only one to mandate perfusion imaging, requiring evidence of salvageable tissue using the automated RAPID software (ischemic core of less than $70 \mathrm{~mL}$ and target mismatch of $>1.2$ on perfusion imaging). This was also the only trial to report a screening $\log -7798$ patients were screened, with $1044(7 \%)$ receiving IVT and 70 (1\%) receiving endovascular therapy. Four hundred ninety-five of 1044 (47\%) of patients treated with IVT were excluded because CTA did not demonstrate a large artery occlusion. It was estimated that $25 \%$ of clinically eligible patients for thrombectomy were excluded on the basis of perfusion imaging alone. All randomized patients received IVT and endovascular treatment commenced within $6 \mathrm{~h}$. This highly selected cohort translated into excellent outcomes - the reperfusion rate was impressive (mTICI $2 \mathrm{~b} / 3$ 86\%) and this trial had the largest effect size, with $71 \%$ of patients in the intervention group achieving functional independence (mRS 0-2) at 90 days, compared to $40 \%$ in the control arm $(p=0.01$, relative risk, RR 1.8). Furthermore, this trial demonstrated a large trend to mortality benefit (20 to $9 \%$ ), although unlike 
the ESCAPE trial the difference was not statistically significant (RR 0.4, 95\% CI 0.1-1.5), probably due to the small sample size.

In SWIFT-PRIME, conducted in 39 United States and European sites between December 2012 to November 2014, the initial premise was to select a target-mismatch penumbra profile (core $<50 \mathrm{ml}$, ischemic tissue with time to the peak of the residual function, Tmax $>10 \mathrm{~s}$ $<100 \mathrm{ml}$, mismatch volume $>15 \mathrm{ml}$ and mismatch ratio $>1.8$ ) using the automated RAPID penumbral imaging software. However, perfusion mismatch was a requirement in only 71 patients before the protocol was changed to one identifying small core infarcts (NCCT ASPECTS >5) in the next 125 patients. All patients received IVT and the endovascular procedure had to start within $6 \mathrm{~h}$. This trial had the highest rate of reperfusion (mTICI $2 \mathrm{~b} / 3$ 88\%) and demonstrated improvement in functional independence (mRS $0-2)$ at 90 days with $60 \%$ in the intervention group versus $36 \%$ in controls (RR 1.7, 95\% CI 1.23-2.33, $p<0.001$ ).

REVASCAT was the last of the five key studies published in 2015 and randomized patients from Nov 2012 to December 2014 from four centres in Catalonia, Spain. A longer time window was allowed (endovascular procedure to start within $8 \mathrm{~h}$ of stroke onset) and patients were recruited either due to contraindication to IVT (32\%), or lack of revascularization 30 min after tPA infusion. The trial was stopped after the first planned interim analysis when only $25 \%$ of patients had completed 90 days of follow-up. Although the pre-specified boundaries for stopping were not met the steering committee elected to stop the trial due to lack of equipoise following the results of the other positive RCTs. REVASCAT included patients with an NIHSS $\geq 6$ with proven vessel occlusion of the intracranial ICA, MCA or M1 trunk; tandem proximal ICA/MCA-M1 occlusions were allowed. This trial used the highest cut-off on ASPECTS score for inclusion (ASPECTS $>6$ ) but also demonstrated the challenges around using this score to exclude patients - the core lab would have excluded $25 \%$ of participants with ASPECTS $\leq 6$ and a further $9 \%$ who had occlusion of a single M2 only. REVASCAT had the longest door-to-groin puncture time of $269 \mathrm{~min}$. Nevertheless, there were still positive results: the primary outcome demonstrated a common odds ratio of improvement in the distribution of the mRS score of 1.7 (95\% CI 1.05-2.8) and the proportion with good outcome (mRS 0-2) increasing from 28 to 44\% (adjusted odd ratio $2.1,96 \%$ CI 1.1 to 4.0 ).

The THRACE (THRombectomie des Artères CErebrales) trial was the most recently published RCT appearing online in August 2016. Patients were recruited from June 2010 to February 2015 from 26 centres in France. This trial compared IVT within $4 \mathrm{~h}$ (changed from $3 \mathrm{~h}$ after 14 May 2011 and 80 patients enrolled) to IVT within $4 \mathrm{~h}$ plus mechanical thrombectomy starting within $5 \mathrm{~h}$, the shortest time window of all trials. Included patients had NIHSS 10-25 and proximal vessel occlusion. The trial included over 400 patients making it the second largest thrombectomy trial behind MR CLEAN $(n=500)$. THRACE resembled IMS with a bridging IV/IA protocol but used the newest stent retrievers and aspiration devices. Importantly imaging assessment of infarct/ischemic extent using ASPECTS or perfusion was not used to exclude patients representing the widest patient selection profile of all RCTs to date. Interestingly, 17/57 (30\%) of patients with ASPECTS $0-4$ had a good clinical outcome. Unlike the other trials that incorporated a drip and ship model, THRACE only included patients presenting directly to an interventional centre, representing a mothership model of care. This trial used functional independence (mRS $0-2)$ at 3 months as the primary outcome and was also a positive trial, with a significantly higher proportion in the mechanical thrombectomy arm reaching this good outcome - 53\% versus 42\%, (OR 1.55 95\% CI 1.05-2.3; $p=0.028$ ). The lower difference between intervention and control group was thought to reflect the longer randomization to groin puncture time of $82 \mathrm{~min}$ which would have adversely affected the intervention arm. The rate of IA reperfusion (TICI 2b-3) was $69 \%$.

\section{Not all recent $R C T$ s have been positive}

THERAPY, using the Penumbra aspiration system rather than a retrievable stent, presented in 2015 and published in September 2016 and PISTE, a pragmatic trial conducted in the UK only, have both reported a trend to improved outcome that did not reach statistical significance $[22,23]$. In an attempt to identify the poorest prognosis patients the THERAPY trial targeted patients with a thrombus length of $8 \mathrm{~mm}$ or longer but failed to show any significant difference in the primary outcome of functional independnece ( $\mathrm{mRS} 0-2$ ) at 90 days, $38 \%$ IAT v $30 \%$ IVT groups $(p=0.52)$.

\section{Efficacy and safety}

The evidence is overwhelmingly in favour of endovascular thrombectomy for large vessel occlusion in patients with acute ischemic stroke. All trials aimed for rapid recanalization, mandated advanced imaging, to greater or lesser degrees, for patient selection, and used newergeneration retrievable stents. These improvements likely resulted in positive effect sizes where previous trials failed. In keeping with this, in a sub-analysis of IMS III, patients with documented occlusion who achieved recanalization within $6 \mathrm{~h}$ had a significant benefit [24]. All trials showed increased functional independence at 90 days with treatment with only one study, ESCAPE, 
showing significantly reduced mortality. Variability in effect size likely relate to slightly different selection criteria, devices used, and speed of treatment.

There was no increase in the rate of intracranial haemorrhage in any of the trials despite concurrent use of thrombolysis for the majority of patients. In the MR CLEAN trial, there was a low rate of distal embolization into new territories (8.6\%), procedure-related dissections $(1.7 \%)$, and vessel perforations $(0.9 \%)$.

\section{Meta-analyses of trials}

Multiple meta-analyses, including various combinations of the three negative RCTs published in 2013 (MR RESCUE, IMS III and SYNTHESIS) with the six positive RCTs published or presented in 2015 (MR CLEAN, ESCAPE, SWIFT-PRIME, EXTEND IA, REVASCAT, THRACE) have confirmed the benefit of endovascular thrombectomy in large vessel occlusion [25-30]. The HERMES collaboration analysed individual patient level data from five of the six positive RCTs published in 2015 (MR CLEAN, ESCAPE, REVASCAT, SWIFT-PRIME and EXTEND-IA; THRACE had not yet been published) [25]. For the primary outcome (the degree of disability on the $\mathrm{mRS}$ ) the adjusted combined odds ratio for reduced disability at 90 days was $2.49,95 \%$ CI 1.76-3.53, $p<0.0001$. The number needed to treat for one patient to have reduced disability of at least 1 point on the mRS was 2.6. The adjusted odds ratio for patients achieving functional independence (mRS 0-2) at 90 days was 2.71 (95\% CI $2.07-3.55 ; p<0.0001)$.

In another meta-analysis, including the negative 2013 RCTs with the same five positive new trials as HERMES, the odds ratio summary statistic for reduction of disability at 90 days was 1.56 ; $95 \%$ CI $1.14-2.13 ; p=0.05$ and the odds ratio for functional independence (mRS 0-2) at 90 days was $1.71(1.18-2.49)$ [26]. The largest meta-analysis to date included the above eight trials but also preliminary data from THERAPY and THRACE, and showed similar benefit with risk ratio for good functional outcome (mRS $0-2)$ at 90 days reported as 1.37; 95\% CI 1.14-1.64 [27]. A subgroup of the seven trials published or presented in 2015, more reflective of current practice with improved patient selection and use of retrievable stents, yielded a risk ratio of 1.56 (95\% CI 1.38-1.75) for a good functional outcome (mRS 0-2) at 90 days. Consistently, all the various meta-analyses showed no significant difference in mortality or intracranial haemorrhage.

Several cost utility and cost effectiveness analyses have suggested that although EVT is a costly procedure it is likely to be cost effective [31-33].

\section{Patient selection - Guidelines and controversies}

Based on the recent positive RCTs, multiple guidelines around the world (eg American Heart Association - AHA, Canadian Stroke Best Practice - CSBP and European Stroke
Organization - ESO) have been developed to synthesize the patient selection criteria and facilitate decision making [34-36]. All now recommend endovascular therapy as the new standard of care for patients with acute ischemic stroke presenting with a large vessel intracranial occlusion (i.e., occlusion of the distal internal carotid artery or proximal middle cerebral artery) identified on vascular imaging, and provide their highest level of recommendation for various other clinical and radiological criteria:

(1)neurologic deficits (CSBP - disabling stroke; AHA - NIHSS $\geq 6$; ESO - not defined)

(2) time (CSBP - within $6 \mathrm{~h}$ with a level B recommendation for patients up to $12 \mathrm{~h}$ based on ESCAPE criteria; AHA - 6 h; ESO - 6 h)

(3) lack of an established infarct i.e., a small core, (CSBP - ASPECTS $\geq 6$; AHA - ASPECTS $\geq 6$; ESO - Grade B, level 2a for "large infarcts may be excluded" but no ASPECTS cut-off suggested)

(4)advanced imaging - Intracranial vascular imaging is strongly recommended for decision making, but the benefits of additional imaging such as MRI or CT perfusion are unknown.

(5) baseline function - the AHA guideline is the only one to explicitly mandate a certain level of baseline function, i.e., a pre-stroke mRS score 0 to 1

(6)intravenous tPA - all recommend treatment with IVT for eligible patients prior to endovascular therapy. However both the CSBP and ESO guidelines maintain their highest level of recommendation for patients ineligible for IVT whereas the AHA reduce their level of recommendation for this subgroup (class IIa, level C)

(7)type of anesthesia - conscious sedation is preferred over general anesthesia (unless medically indicated) by both AHA and CSBP recommendations, albeit with reduced levels of evidence (class IIb level $\mathrm{C}$ and level $\mathrm{B}$ respectively) whereas the ESO guideline leaves this as an individual patient decision. Several RCTs are underway to address this issue [37, 38].

(8)age - an upper age limit is not specified by any of the guidelines with the ESO guideline explicitly stating that "high age alone is not a reason to withhold mechanical thrombectomy", assigning this recommendation Grade A, level 1a evidence.

(9) Tandem occlusions (additional extracranial ICA occlusion): both the CSBP and ESO guidelines do not provide an explicit recommendation for this cohort. However, the AHA guideline states that "angioplasty or stenting of proximal cervical atherosclerotic stenosis or complete occlusion at the time of thrombectomy may be considered but its usefulness is unknown" (Class IIb, Level C) 


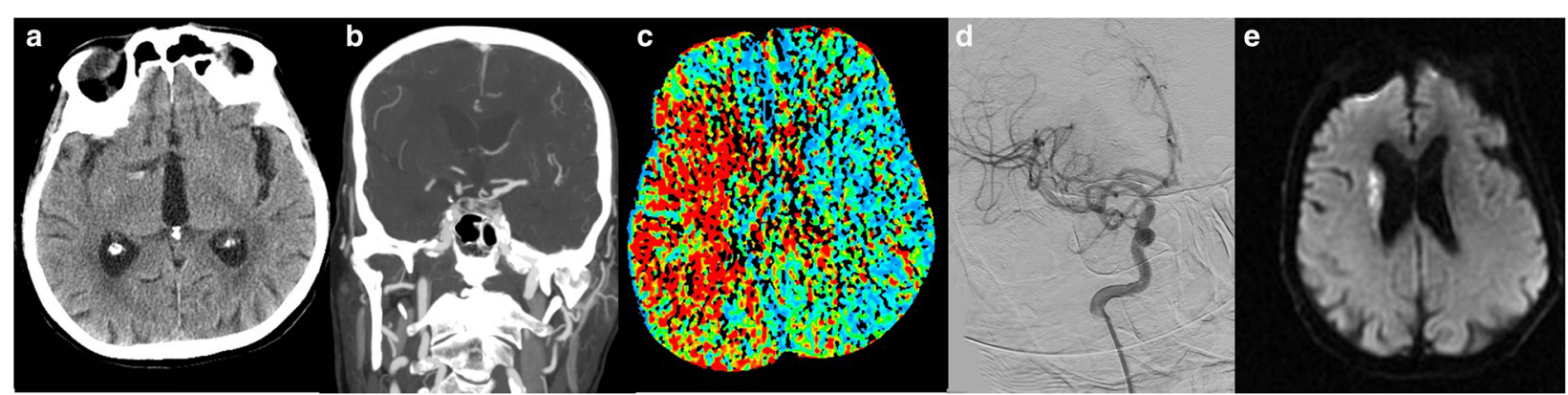

Fig. 1 Illustrative cases of difficult patients pushing boundaries of conventional therapeutic criteria: Advanced age and baseline function. Ninety-five year old male with known dementia - lives at home with his wife, ambulates independently but needs assistance with activities of daily living; pre-stroke mRS 3. Presents with a left MCA syndrome, NIHSS 22. Very early presentation with very favourable imaging - ASPECTS 9 on NCCT (a). Note the hyperdense right proximal MCA. CTA (b) confirmed a left M1 occlusion with a large clot extending along the M1 trunk into the M2 vessels. Good collaterals were present with a large mismatch on CT perfusion (c). The patient was treated with IVT - door to needle (DTN) time 32 min; onset to tPA treatment (OTT) time $74 \mathrm{~min}$. Successful EVT recanalization at 150 min post stroke onset (d). A small deep infarct was present on DWI (e). Patient was discharged home on day 8 with NIHSS of 5 and was ambulating independently

(10)Type of device - The use of retrievable stents is recommended with the highest level of evidence by all three guidelines, with lower levels of recommendations given to consider other devices based on local protocols. A recent analysis of the MR CLEAN data showed that choice of device did not influence outcomes in that data set. The majority were treated with the Trevo device, 53\%, Solitaire device $13 \%$ and other device 17\% [39].

\section{Conclusions}

Endovascular therapy is now the standard of care in patients with large vessel anterior circulation occlusion. This creates challenges in providing care to appropriate patients within a rapid timeframe, since only few highly specialized centres have access to the necessary skills and technology $[40,41]$. Peripheral centres may or may not have rapid access to non-invasive angiographic imaging 24/7 in order to screen stroke patients for large vessel occlusion. Therefore currently decisions to transfer patients to tertiary care centre must be made on the basis of patient presentation and non-contrast head CT, which can be challenging. Whether it is best to build the capacity of peripheral centres in providing vascular imaging (providing more accurate diagnosis by screening out clinical false positives but delaying arrival to endovascular centres), or centralizing acute stroke care by transferring all patients who have a possibility of large vessel occlusion (rapid access for selected patients, but many false negatives will place a large burden on tertiary care centres), must be further determined.

The expanded and varying time window used across the trials creates challenges and opportunities in treating patients with proximal occlusion (Fig. 1). "Wake up strokes," typically deemed out of the time window for thrombolysis given the lack of clear time of onset, may be candidates for endovascular therapy if a proximal occlusion is identified (Fig. 2) [42]. Multi-model imaging, including perfusion, and

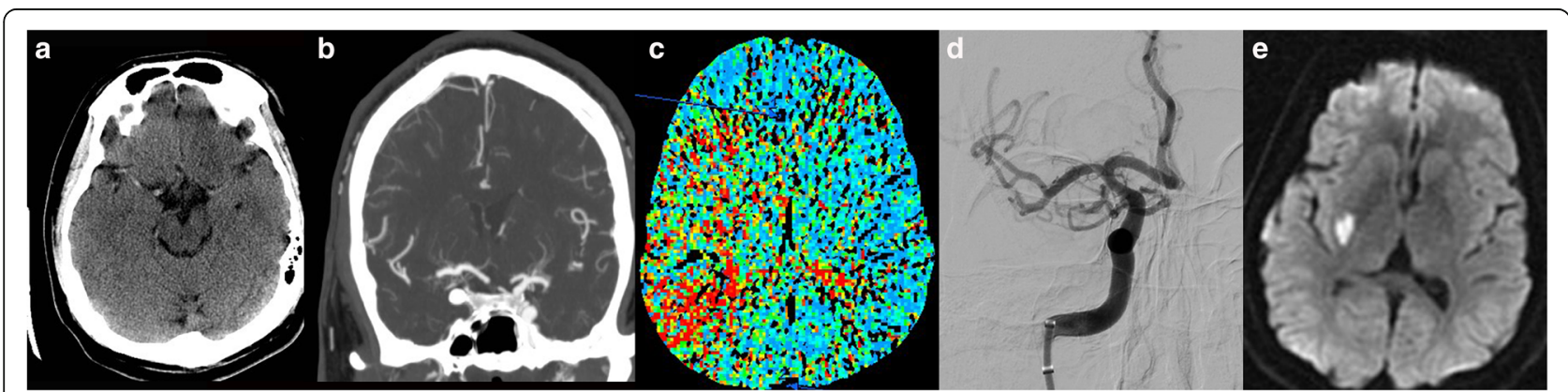

Fig. 2 Illustrative cases of difficult patients pushing boundaries of conventional therapeutic criteria: Unclear time of onset. Twenty-nine year old male last seen normal at 4 am. Found the same day at 1 pm. Presents with right MCA syndrome with severe left sided weakness. NIHSS 8. ASPECTS 10 with hyperdense right MCA (a). CTA reveals right intracranial ICA and mid to distal M1 occlusion with good collaterals (b). Mismatch demonstrated on CT perfusion (c). IVT not given. EVT performed and successful recanalization $13 \mathrm{~h} 29 \mathrm{~min}$ post last seen well time (d). DWI confirmed right deep putamen infarct (e). Patient was discharged home on day 3 with NIHSS 0 


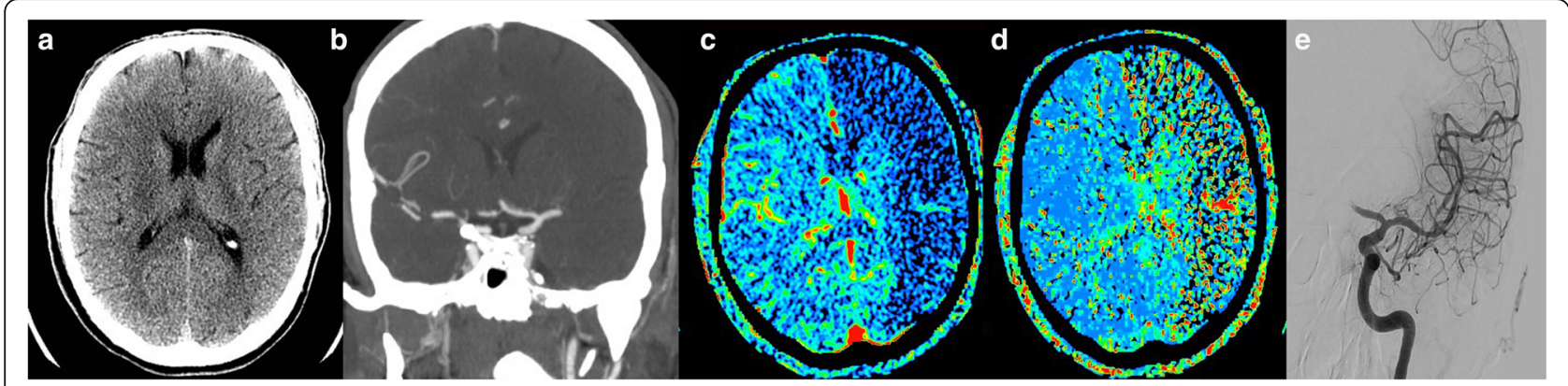

Fig. 3 Illustrative cases of difficult patients pushing boundaries of conventional therapeutic criteria: Poor collaterals but early time to presentation and treatment. Forty-two year old male with witnessed collapse at 19:20. Presents ultra-early, 35 min post collapse, with left MCA syndrome including right sided weakness, forced gaze deviation and mutism with NIHSS 22. Unfavorable imaging including ASPECTS 5 (a), hyperdense left MCA vessel, left M1 (CTA b), left proximal A2 (not shown) occlusions with poor collaterals and matched CT perfusion defect (CBV, MTT c, d). Patient was treated with IVT (DTN time $37 \mathrm{~min}$; OTT time $72 \mathrm{~min}$ ) and EVT (DSA (e) with successful recanalization 141 min post stroke onset). No tPA/EVT complications but no neurological improvement with a $24 \mathrm{~h}$ NIHSS of 23. Patient developed malignant infarction requiring decompressive hemicraniectomy. Day 90 mRS 2

assessment of collaterals may help identify patients who do not yet have established infarct and who may still benefit from reperfusion despite unknown time of onset [43]. Multimodal imaging has the potential to remove strict time windows on delivering therapy, but rather focus on individualizing therapy to patients with salvageable brain tissue. The role of collaterals in patient selection and outcome remains to be determined (Fig. 3).

The new trials were conducted in patients with occlusion in the ICA, M1, proximal M2, A1, and proximal A2. More distal clots were not adequately assessed in the recent trials and occlusions of the posterior circulation eg basilar artery occlusions, were not even included. Further trials are needed to address the feasibility, efficacy, and safety of extracting clots from the distal M2 or M3 or the posterior circulation. The newer generation retrievable stents, Solitaire and Trevo, have demonstrated superiority in recanalization and clinical outcomes, compared to older generation devices and further technological developments may be geared to reducing complications such as distal embolization.

The future is bright for acute treatment in ischemic stroke but much work remains to be done.

\section{Abbreviations}

A1: First portion of anterior cerebral artery; A2: Second portion of anterior cerebral artery; AHA: American Heart Association; AIS: Acute ischemic stroke; ASPECTS: Alberta Stroke Program Early CT Score; Bridging: IV tPA + thrombectomy; CBF: Cerebral blood flow; CSBP: Canadian stroke best practice; CT: Computed tomography; CTA: Computer tomography angiography; CTP: Computed tomography perfusion; ESO: European Stroke Organization; EVT: Endovascular thrombectomy; IAT: Intra-arterial thrombolysis; I-ICA: Intracranial internal carotid artery; IVT: Intravenous thrombolysis; M1: First portion of the middle cerebral artery, from the origin to the bifurcation/trifurcation; M2: Second portion of the middle cerebral artery; mAOL: modified arterial occlusion lesion score; MCA: Middle cerebral artery; mRS: modified Ranking Scale; mTICl: modified thrombolysis in cerebral infarction score; NIHSS: National Institutes of Health Stroke Scale; nr: not recorded; PH-2: Parenchymal hematoma 2 , ie. $>30 \%$ of the infarcted area with significant space-occupying effect, or clot remote from infarcted area; RCT: Randomized control trial; RR: Relative risk; SAE: Serious adverse events; sICH: symptomatic intracranial haemorrhage; TIMI: Thrombolysis in myocardial infarction score; Tmax: Time to the peak of the residual function; tPA: tissue plasminogen activator; VICBV: very low cerebral blood volume

\section{Acknowledgements}

Not applicable.

\section{Funding}

Dr Aviv is funded by CIHR Project Grant 148672. The funding agency played no role in the design of the study and collection, analysis, and interpretation of data or in writing the manuscript.

\section{Availability of data and materials}

Data sharing not applicable to this article as no datasets were generated or analysed during the current study.

\section{Authors' contributions}

$K B$ was the primary author. All authors (KB,RAJ,RIA) contributed to the literature review, manuscript writing, review and editing. All authors read and approved the final manuscript.

\section{Competing interests}

The authors declare that they have no competing interests.

\section{Consent for publication}

Not applicable.

\section{Author details}

'Department of Medicine, Division of Neurology, Sunnybrook Health Science Centre and University of Toronto, 2075 Bayview Avenue, Toronto M4N 3M5, Canada. ${ }^{2}$ Department of Medical Imaging, Division of Neuroradiology,

Sunnybrook Health Science Centre and University of Toronto, 2075 Bayview Avenue, Toronto M4N 3M5, Canada.

Received: 28 November 2016 Accepted: 8 December 2016 Published online: 27 February 2017

\section{References}

1. The National Institute of Neurological Disorders and Stroke rt-PA Study Group. Tissue plasminogen activator for acute ischemic stroke. N Engl J Med. 1995;333:1581-7. 2.

2. Hacke W, Kaste M, Bluhmki E, et al. Thrombolysis with alteplase 3 to 4.5 hours after acute ischemic stroke. NEJM. 2008;359:1317-29.

3. Emberson J, Lees K, Lyden P, et al. Effect of treatment delay, age and stroke severity on the effects of intravenous thrombolysis with alteplase for acute ischaemic stroke: a meta-analysis of individual patient data from randomised trials. Lancet. 2014;384:1929-35. 
4. Del Zoppo G, Higashida R, Furlan A, et al. PROACT: a phase II randomized trial of recombinant Pro-urokinase by direct arterial delivery in acute middle cerebral artery stroke. Stroke. 1998;29:4-11.

5. Furlan A, Higashida R, Wechsler L, et al. Intra-arterial prourokinase for acute ischemic stroke - the PROACT II study: a randomized controlled trial. JAMA. 1999;282:2003-11.

6. Smith W, Sung G, Starkman S, et al. Safety and efficacy of mechanical embolectomy in acute ischemic stroke: results of the $\mathrm{MERCI}$ trial. Stroke. 2005;36:1432-8

7. Smith W, Sunf G, Saver J, et al. Mechanical thrombectomy for acute ischemic stroke: final results of the multi MERCI trial. Stroke. 2008;39:1205-12.

8. The Penumbra Pivotal Stroke Trial Investigators. The penumbra pivotal stroke trial: safety and effectiveness of a New generation of mechanical devices for clot removal in intracranial large vessel occlusive disease. Stroke. 2009;40:2761-8.

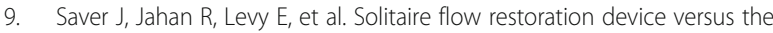
Meric Retriever in patients with acute ischaemic stroke (SWIFT): a randomized parallel-groip, non-inferiority trial. Lancet. 2012;380:1241-9.

10. Nogueira R, Lutsep H, Gupta R, et al. Trevo versus Merci retrievers for thrombectomy revascularisation of large vessel occlusions in acute ischaemic stroke (TREVO 2): a randomised trial. Lancet. 2012;380:1231-40.

11. Kidwell CS, Jahan R, Gornbein J, et al. MR RESCUE Investigators. A trial of imaging selection and endovascular treatment for ischemic stroke. N Engl J Med. 2013;368:914-23.

12. Broderick JP, Palesch YY, Demchuk AM, et al. Interventional Management of Stroke (IMS) III Investigators. Endovascular therapy after intravenous t-PA versus t-PA alone for stroke. N Engl J Med. 2013;368:893-903.

13. Ciccone A, Valvassori L, Nichelatti M, et al. SYNTHESIS expansion investigators. Endovascular treatment for acute ischemic stroke. N Engl J Med. 2013;368:904-13.

14. Goyal M, Almekhlafi MA, Fan L, et al. Evaluation of interval times from onset to reperfusion in patients undergoing endovascular therapy in the Interventional Management of Stroke III trial. Circulation. 2014;130:265-72.

15. Menon BK, Almekhlafi MA, Pereira VM, et al. Optimal workflow and processbased performance measures for endovascular therapy in acute ischemic stroke: analysis of the Solitaire FR Thrombectomy for Acute Revascularization study. Stroke. 2014;45:2024-9.

16. Berkhemer $\mathrm{O}$, Beumer $F$, van den Berg $L$, et al. A randomized trial of intraarterial treatment for acute ischemic stroke (MR CLEAN). NEJM. 2015:372:11-20.

17. Goyal M, Demchuk A, Menon B. Randomized assessment of rapid endovascular treatment of ischemic stroke (ESCAPE). NEJM. 2015;372:1019-30

18. Campbell B, Mitchell P, Kleinig T, et al. Endovascular therapy for ischemic stroke with perfusion-imaging selection (EXTEND-IA). NEJM. 2015;372:1009-18.

19. Saver J, Goyal M, Bonafe A, et al. Stent-retriever thrombectomy after intravenous t-PA vs. t-PA alone in stroke (SWIFT-PRIME). NEJM. 2015;372:2285-95.

20. Jovin T, Chamorro A, Cobo E, et al. Thrombectomy within 8 hours after symptom onset in ischemic stroke (REVASCAT). NEJM. 2015;372:2296-306.

21. Baccard S, Ducrocq X, Mas J, et al. Mechanical thrombectomy after intravenous alteplase versus alteplase alone after stroke (THRACE): a randomized controlled trial. Lancet Neurol. 2016;15:1138-47.

22. Mocco J, Osama O. Zaidat, Rüdiger von Kummer, et al. Aspiration Thrombectomy After Intravenous Alteplase Versus Intravenous Alteplase Alone (THERAPY). Stroke 2016;47(9):2331-8

23. Muir k, Ford G, Messow C et al. Endovascular therapy for acute ischaemic stroke: the Pragmatic Ischaemic Stroke Thrombectomy Evaluation (PISTE) randomised, controlled trial. JNNP 2016 Oct 18. doi: 10.1136/jnnp-2016314117 (Epub ahead of print)

24. Demchuk AM, Goyal M, Yeatts SD, et al. Recanalization and clinical outcome of occlusion sites at baseline CT angiography in the Interventional Management of Stroke III trial. Radiology. 2014;273:202-10.

25. Goyal M, Menon B, van Zwam W, et al. Endovascular thrombectomy after large-vessel ischaemic stroke: a meta-analysis of individual patient data from five randomized controlled trials, (HERMES collaboration). Lancet. 2016;387:1723-31.

26. Badhiwala J, Nassiri F, Alhazzani W, et al. Endovascular thrombectomy for acute ischemic stroke - a meta-analysis. JAMA. 2015;314(17):1832-43.

27. Rodrigues F, Neves J, Caldeira D, et al. Endovascular treatment versus medical care alone for ischaemic stroke: systematic review and metaanalysis. BMJ. 2016;353:i1754. doi:10.1136/bmj.i1754.
28. Balami J, Sutherland B, Edmunds L, et al. A systematic review and metaanalysis of randomized controlled trials of endovascular thrombectomy compared with best medical treatment for acute ischemic stroke. Int J Stroke. 2015:10:1168-78.

29. Elgendy I, Kumbhani D, Mahmoud A, et al. Mechanical thrombectomy for acute ischemic stroke - a meta-analysis of randomized trials. J Am Coll Cardiol. 2015;66:2498-505.

30. Sardar P, Chatterjee S, Giri J, et al. Endovascular therapy for acute ischaemic stroke: a systematic review and meta-analysis of randomized trails. Eur Heart J. 2015. doi:10.1093/eurheartj/ehv270.

31. Ganesalingam J, Pizzo E, Morris S, et al. Cost-utility analysis of mechanical thrombectomy using stent retrievers in acute ischemic stroke. Stroke. 2015;46:2591-8.

32. Leppert M, Campbell J, Simpson J, et al. Cost-effectiveness of intra-arterial treatment as an adjunct to intravenous tissue-type plasminogen activator for acute ischemic stroke. Stroke. 2015;46:1870-6.

33. Xie X, Lambrinos A, Chan B, et al. Mechanical thrombectomy in patients with acute ischemic stroke: a cost-utility analysis. CMAJ Open. 2016. doi:10.9778/cmajo.20150088.

34. Powers WJ, Derdeyn CP, Biller J, Ameri- can Heart Association Stroke Council, et al. 2015 AHA/ASA focused update of the 2013 guidelines for the early management of patients with acute ischemic stroke regarding endovascular treat- ment: a guideline for healthcare professionals from the American Heart Association/American Stroke Association. Stroke 2015;46(10): 3020-35.

35. Casaubon LK, Boulanger J-M, Blacquiere D, et al. Heart and Stroke Foundation of Canada Canadian Stroke Best Practices Advisory Committee. Canadian stroke best practice recommendations: hyper- acute stroke care guidelines, update 2015. Int J Stroke. 2015;10:924-40.

36. Wahlgren N, Moreira T, Michel P, et al. Mechanical thrombectomy in acute ischemic stroke: Consensus statement by ESO-Karolinksa Stroke Update 2014/ 2015, supported by ESO, ESMINT, ESNR and EAN. Int Stroke. 2016;11(1):134-47.

37. Sedation vs. Intubation for Endovascular Stroke Treatment (SIESTA) ClinicalTrials.gov NCT02126085.

38. General Anesthesia Versus Sedation During Intra-arterial Treatment for Stroke (GASS). ClinicalTrials.gov NCT02822144

39. Dippel D, Majoie C, Roos Y, et al. Influence of device choice on the effect of intra-arterial treatment for acute ichemic stroke in MR CLEAN. Stroke. 2016:47:2574-81.

40. Fisher M, Saver JL. Future directions of acute ischaemic stroke therapy. Lancet Neurol. 2015;14(7):758-67.

41. Goyal M, Hill MD, Saver JL, et al. Challenges and opportunities of endovascular stroke therapy. Ann Neurol. 2015;79(1):11-7.

42. Trevo and Medical Management Versus Medical Management Alone in Wake-Up and Late Presenting Strokes (DAWN, ClinicaTrials.gov NCT02142283)

43. Perfusion Imaging Selection of Ischemic Stroke Patients for Endovascular Therapy (POSITIVE, ClinicalTrials.gov NCT01852201) 